\title{
Plasma Pentraxin 3 is a More Potent Predictor of Endothelial Dysfunction than High-Sensitive C-Reactive Protein
}

\author{
Tomohiko Yasunaga, ${ }^{1}$ MD, Satoshi IKeda, ${ }^{1}$ MD, Seiji Koga, ${ }^{1}$ MD, Tomoo NaKata,,${ }^{1}$ MD, \\ Takeo Yoshida, ${ }^{1}$ MD, Nobuhito Masuda, ${ }^{2} \mathrm{PhD}$, Shigeru Kohno, ${ }^{3} \mathrm{MD}$, and Koji Maemura, ${ }^{1} \mathrm{MD}$
}

\begin{abstract}
SUMMARY
An inflammatory response is a key event for endothelial dysfunction. Pentraxin 3 (PTX3) is an inflammatory protein produced at inflammation sites such as leukocytes and vascular endothelial cells. Here, we compared the relationships between endothelial function assessed by flow-mediated dilation (FMD), and the levels of plasma PTX3 and highsensitive C-reactive protein (hsCRP), another inflammatory protein of the pentraxin family.

Levels of FMD, PTX3 and hsCRP were measured twice within 6 to 8 months and retrospectively analyzed in 36 patients with coronary artery disease. We examined the associations between the values of FMD and the levels of PTX3 and hsCRP at the first measurement, and between the change ratios (second value/first value) of these parameters.

Univariate linear regression analysis showed significantly negative correlations between FMD values and PTX3 and hsCRP levels at the first measurement, and significant associations with taking statins or calcium antagonists. Multivariate linear stepwise regression analysis identified PTX3 levels and taking statins and calcium antagonists as independent factors for endothelial function. The change ratio of FMD correlated more closely with that of PTX3 than of hsCRP ( $r=-0.446, P=0.006$ versus $r=-0.330, P=0.050$ ). Significantly more patients with decreased FMD values had increased levels of PTX3 than those of hsCRP at the second measurement compared with the first measurement. Furthermore, the ratio of patients with increased PTX3, but not increased hsCRP, was significantly reduced among those with increased, rather than decreased, FMD values.
\end{abstract}

Endothelial dysfunction might be more accurately predicted by plasma PTX3 levels than by serum hsCRP levels. (Int Heart J 2014; 55: 160-164)

Key words: Inflammation, Flow mediated dilation, Coronary artery disease

$\mathrm{T}$ The vascular endothelium plays a key role in the control of vasomotor tone, platelet adhesion, and thrombosis through the release of nitric oxide (NO), prostaglandins, and other vasoactive compounds. ${ }^{1,2)}$ Endothelial dysfunction is considered to be an early pathophysiological feature of atherosclerosis and an independent predictor of cardiovascular diseases. ${ }^{3)}$ Therefore, assessment of endothelial function is important, especially for patients at risk for coronary artery diseases (CAD). Flow-mediated dilation (FMD) is widely used for assessing endothelial function. FMD is determined by noninvasive ultrasound assessment of hyperemia-induced responses at the brachial artery, and is presently regarded as a promising tool for the prediction of CAD risk. ${ }^{3-5)}$

An inflammatory response represents the key piece of evidence leading to endothelial dysfunction and the subsequent formation of atheromatous plaques. ${ }^{1,6)} \mathrm{C}$-reactive protein (CRP), a member of the pentraxin family, is the most well-established inflammation marker and provides independent prognostic information in relation to various coronary heart diseases. ${ }^{7)}$ CRP levels have been reported to be inversely correlated with endothelial vasoreactivity. ${ }^{8)}$ Pentraxin 3 (PTX3), a member of the long pentraxin family, is rapidly induced in various cell subsets, including leukocytes, myeloid dendritic cells, and vascular endothelium under stimulation with inflammatory cytokines. ${ }^{9)}$ In contrast to CRP, which is primarily synthesized in the liver and thus reflects systemic inflammation, PTX3 is synthesized locally, in the vascular system. ${ }^{10)}$ Therefore, PTX3 levels are thought to reflect endothelial dysfunction. However, this relationship has yet to be clarified. Furthermore, the relationship between endothelial function and PTX3, as well that between endothelial function and CRP, also has yet to be clarified. The present study thus investigated both whether plasma PTX3 levels are associated with endothelial function in patients with $\mathrm{CAD}$, and whether differences can be seen in the association between PTX3 and CRP.

\section{Methods}

Patients: A total of 36 patients with CAD were retrospectively

From the ${ }^{1}$ Department of Cardiovascular Medicine, Nagasaki University Graduate School of Biomedical Sciences, Nagasaki, ${ }^{2}$ Perseus Proteomics Inc., Tokyo, and ${ }^{3}$ Second Department of Internal Medicine, Nagasaki University School of Medicine, Nagasaki, Japan.

Address for correspondence: Satoshi Ikeda, MD, Department of Cardiovascular Medicine, Nagasaki University Graduate School of Biomedical Sciences, 1-7-1 Sakamoto, Nagasaki, Nagasaki 852-8501, Japan. E-mail: sikeda@nagasaki-u.ac.jp

Received for publication August 25, 2013. Revised and accepted September 17, 2013

Released advance online J-STAGE March 14, 2014.

All rights are reserved to the International Heart Journal Association. 
recruited for this study. Levels of FMD, PTX3, and high-sensitive CRP (hsCRP) were measured twice between December 2008 and April 2010 at Nagasaki University Hospital. Based on the classification of the American Heart Association, the presence of CAD was defined as angiographic evidence of $\geq$ $75 \%$ stenosis in at least one coronary artery, and/or a history of percutaneous coronary intervention and/or coronary artery bypass grafting or myocardial infarction. Exclusion criteria included the following: having clinically unstable features such as acute coronary syndrome or New York Heart Association class III or IV heart failure; having stage 5 chronic kidney disease, which included being on hemodialysis; and a history of treatment for or diagnosis of carotid artery stenosis, thoracic/ abdominal aortic aneurysm, peripheral artery disease, collagen disease, malignancy, infections, or other systemic inflammatory diseases.

FMD and blood profiles, including plasma PTX3, lipid panel, hemoglobin A1c ( $\mathrm{HbA} 1 \mathrm{c})$ and renal function, were assessed twice within 6 to 8 months $(203.5 \pm 18.4$ days). Hypertension was defined as either being treated with antihypertensive agents or having systolic blood pressure of $\geq 140 \mathrm{mmHg}$ and/or diastolic blood pressure of $\geq 90 \mathrm{mmHg}$. Diabetes was defined as having fasting blood glucose $\geq 126 \mathrm{mg} / \mathrm{dL}, \mathrm{HbA} 1 \mathrm{c}$ $[\mathrm{NGSP}] \geq 6.5 \%$ or being medicated with antidiabetes drugs. The value for HbA1c (\%) is estimated as an NGSP equivalent value $(\%)$ calculated using the following formula:

HbA1c $(\%)=$ HbAlc (JDS) $(\%)+0.4 \%$,

where the relational expression of HbA1c (JDS) (\%) is measured using the previous Japanese standard substance and measurement methods and HbA1c (NGSP). Dyslipidemia was defined as being medicated with lipid lowering agents, or as having low-density lipoprotein cholesterol (LDL-cholesterol) $\geq$ $140 \mathrm{mg} / \mathrm{dL}$, triglycerides $\geq 150 \mathrm{mg} / \mathrm{dL}$, and/or high-density lipoprotein cholesterol (HDL-cholesterol) $<40 \mathrm{mg} / \mathrm{dL}$.

This study complied with the principles of the Declaration of Helsinki in regard to human investigations, and its protocol was approved by the Ethics Committee of Nagasaki University Hospital. Written, informed consent was obtained from all participants before enrollment in the study.

Measurement of brachial arterial FMD: Brachial arterial FMD was assessed using a novel vascular ultrasound system equipped with both an edge-tracking system for 2D imaging and a pulsed Doppler flow velocimeter for automatic measurement (UNEXEF, Unex Co. Ltd., Nagoya, Japan). Patients were examined between 7:00 and 8:00 AM in the morning after being instructed to fast and abstain from caffeinated beverages, tobacco products, and vitamin supplements for 12 hours prior to the investigations. They were also instructed to abstain from exercise after waking up until the end of the examination. All vasoactive medications were withheld for at least 12 hours. To allow consistent recording of the brachial artery 2 to $4 \mathrm{~cm}$ above the antecubital fossa, all patients remained at rest in a supine position with the right arm extended and comfortably immobilized. As described by Celemajer, et al, ${ }^{11,12)}$ longitudinal scans of the brachial artery were then acquired, and FMD was induced by inflating a pneumatic tourniquet that had been placed on the forearm to a pressure $>200 \mathrm{mmHg}$ for $5 \mathrm{~min}$ utes. After the cuff was released, serial images of the brachial artery were acquired for 5 minutes, during which time the diameter of the brachial artery was continuously measured. FMD was defined as the ratio (\%) change in arterial diameter at one minute after cuff deflation compared with baseline resting diameter.

Measurement of blood samples: Venous blood samples were withdrawn from the forearms of all patients who had fasted overnight. Total LDL- and HDL-cholesterol, triglyceride, creatinine, and $\mathrm{HbA} 1 \mathrm{c}$ levels were measured at our hospital using routine laboratory techniques. Venous blood was collected into EDTA vacuum containers and stored at $-80^{\circ} \mathrm{C}$. PTX3 levels were then measured using a high-sensitivity, enzyme-linked immunosorbent assay system for human plasma (Perseus Proteomics, Tokyo). High-sensitive CRP was measured at SRL Co. Ltd. (Tokyo). The estimated glomerular filtration rate (eGFR) was calculated as follows: $194 \times$ age $\mathrm{a}^{-0.287} \times$ serum creatinine ${ }^{-1.094}$ (if female, $\times 0.739$ ). ${ }^{13)}$ Ratios of changes in parameter values between the first and the second measurements were calculated as the value at the second measurement/the value at the first measurement. Values that were higher or lower at the second than at the first measurement were defined as a respective increase or decrease.

Statistical analysis: Continuous values are expressed as the mean \pm standard deviation (SD) and were tested for normal distribution using the Kolmogorov-Smirnov test. Non-normal distributed values, including PTX3, hsCRP, and NT-proBNP, were log-transformed before analysis. Relationships between clinical variables and FMD were evaluated using univariate linear regression analysis, and variables that correlated with FMD $(P<0.10)$ were tested for independence using multivariate stepwise linear regression analysis. Relationships among change ratios of variables were evaluated using Spearman's rank correlation coefficient. Data presented as numbers of patients were analyzed using the chi-square test. A $P$ value $<0.05$ was considered statistically significant. All other data were statistically analyzed using SPSS version 18 (IBM Corp., Somers, NY).

\section{RESULTS}

Table I shows the characteristics of the patients at the first FMD measurement. The patients were predominantly male, relatively older (mean, 68.5 years) and had a normal mean body mass index. Comorbid conditions included diabetes mellitus $(44.4 \%)$, hypertension $(80.6 \%)$, and dyslipidemia $(61.1 \%)$, and $69.4 \%$ were smokers.

Univariate linear regression analysis showed that FMD values significantly and negatively correlated with log-PTX3 and log-hsCRP levels at the first examination (Table II and Figure 1). Medication with calcium antagonists and statins also correlated with FMD values (Table II). Multivariate stepwise linear regression analysis, in which variables with a $P$ value < 0.10 in univariate analysis were incorporated into the model, identified log-PTX3 and medication with calcium antagonists and statins as independent factors associated with FMD.

The ratio of change in FMD between the first and the second measurement significantly correlated with that of logPTX3 and log-hsCRP (Table III, Figures 2A and B), and the correlation with log-PTX3 was closer than that with loghsCRP $(r=-0.446, P=0.006$ versus $r=-0.330, P=0.050$, respectively). More patients with decreased FMD had increased PTX3 compared with increased hsCRP $(P=0.049$; Figure $2 \mathrm{C})$. Furthermore, the ratio of patients with increased PTX3, 
but not increased hsCRP, was significantly reduced among those with increased, rather than decreased, FMD values $(P=$

Table I. Characteristics of the Participants

\begin{tabular}{|c|c|}
\hline Parameters & Mean \pm SD \\
\hline Gender (male:female) & $31: 5$ \\
\hline Age (years) & $68.5 \pm 8.3$ \\
\hline Body mass index $\left(\mathrm{kg} / \mathrm{m}^{2}\right)$ & $23.9 \pm 3.4$ \\
\hline Smoking (\%) & 69.4 \\
\hline Diabetes mellitus (\%) & 47.2 \\
\hline Hypertension (\%) & 80.6 \\
\hline Dyslipidemia (\%) & 61.1 \\
\hline Systolic blood pressure $(\mathrm{mmHg})$ & $127.1 \pm 15.7$ \\
\hline Diastolic blood pressure $(\mathrm{mmHg})$ & $68.0 \pm 6.7$ \\
\hline PTX3 (pg/mL) & $3.18 \pm 1.38$ \\
\hline hsCRP (mg/L) & $4.84 \pm 8.35$ \\
\hline $\mathrm{eGFR}\left(\mathrm{mL} / \mathrm{min} / 1.73 \mathrm{~m}^{2}\right)$ & $60.8 \pm 21.1$ \\
\hline Total cholesterol (mg/dL) & $172.0 \pm 26.4$ \\
\hline LDL-cholesterol (mg/dL) & $100.9 \pm 23.1$ \\
\hline HDL-cholesterol (mg/dL) & $43.3 \pm 11.1$ \\
\hline Triglyceride (mg/dL) & $127.8 \pm 61.6$ \\
\hline Fasting blood glucose (mg/dL) & $107.4 \pm 26.6$ \\
\hline HbAlc (\%) & $6.42 \pm 0.94$ \\
\hline NT-proBNP (pg/mL) & $583.04 \pm 994.6$ \\
\hline \multicolumn{2}{|l|}{ Medicines } \\
\hline Calcium antagonists (\%) & 50.0 \\
\hline ARB/ACE-I (\%) & 72.2 \\
\hline$\beta$-Blocker $(\%)$ & 16.7 \\
\hline$\alpha$-Blocker $(\%)$ & 2.8 \\
\hline Diuretics $(\%)$ & 8.3 \\
\hline Statin $(\%)$ & 47.2 \\
\hline FMD $(\%)$ & $5.01 \pm 2.11$ \\
\hline
\end{tabular}

ARB indicates angiotensin II receptor blocker; ACE-I, angiotensin converting enzyme inhibitor; A1c; protein; eGFR, estimated glomerular filtration; FMD, flow mediated dilation; HbAlc, hemoglobin A1c protein; HDL-cholesterol, high-density lipoprotein cholesterol; hsCRP, high-sensitive C-reactive protein; LDL-cholesterol, low-density lipoprotein cholesterol; NT-proBNP, N-terminal-pro-B type brain natriuretic peptide; and PTX3, pentraxin 3.
0.003; Figure 2C).

\section{Discussion}

Endothelial dysfunction is an initial step in the atherogenic process. ${ }^{14)}$ It is characterized by the following: increased endothelial cell permeability; a pro-coagulant state; enhanced leukocyte adhesion due to increased endothelial expression of adhesion molecules; increased vascular tone due to a reduction in NO production; and the proliferation of smooth muscle cells. ${ }^{15)}$ FMD is a repeatable, reproducible, and noninvasive ultrasound assessment of endothelial function that closely correlates with invasively measured endothelial function ${ }^{11)}$ and predicts risk of both future fatal and nonfatal cardiovascular events. $^{16}$

An inflammatory response is a key contributing factor to endothelial dysfunction. A significant relationship between CRP and endothelial dysfunction has been determined, showing that CRP impairs both bioactivity in vitro and in vivo and endothelial vasoreactivity in vivo through the inhibition of en-
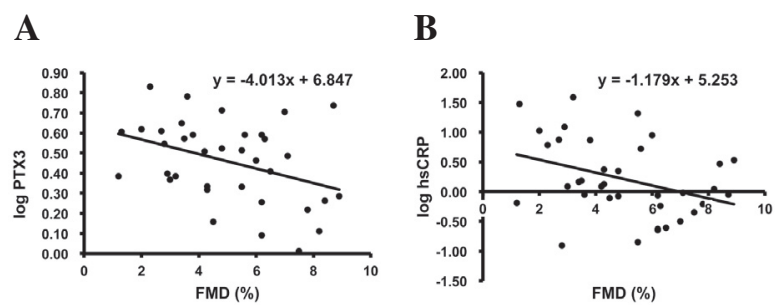

Figure 1. Correlations between flow mediated dilation (FMD) values and both pentraxin 3 (PTX3) and high sensitive C-reactive protein (hsCRP) levels at first examination. FMD values negatively correlate with both log PTX3 (A) and log hsCRP (B) levels. FMD indicates flow mediated dilation; hsCRP, high sensitive C-reactive protein; and PTX3, pentraxin 3.

Table II. Univariate and Multivariate Linear Regression Analyses of Flow Mediated Dilation (FMD)

\begin{tabular}{|c|c|c|c|c|c|}
\hline \multirow[b]{2}{*}{ Variables } & \multicolumn{2}{|c|}{ Univariate } & \multicolumn{3}{|c|}{ Multivariate } \\
\hline & $r$ & $P$ & $\beta$ & SE & $P$ \\
\hline Age (years) & 0.222 & 0.193 & - & - & NS \\
\hline Body mass index $\left(\mathrm{kg} / \mathrm{m}^{2}\right)$ & -0.159 & 0.354 & - & - & NS \\
\hline Systolic blood pressure (mmHg) & -0.332 & 0.052 & - & - & NS \\
\hline Diastolic blood pressure (mmHg) & -0.282 & 0.101 & - & - & NS \\
\hline Log PTX3 (pg/mL) & -0.381 & 0.022 & -0.352 & 1.416 & 0.015 \\
\hline Log hs-CRP (mg/L) & -0.358 & 0.032 & - & - & NS \\
\hline eGFR $\left(\mathrm{mL} / \mathrm{min} / 1.73 \mathrm{~m}^{2}\right)$ & -0.070 & 0.687 & - & - & NS \\
\hline Total cholesterol (mg/dL) & -0.201 & 0.239 & - & - & NS \\
\hline LDL-cholesterol (mg/dL) & -0.297 & 0.078 & - & - & NS \\
\hline HDL-cholesterol (mg/dL) & 0.006 & 0.970 & - & - & NS \\
\hline Log Triglyceride (mg/dL) & 0.128 & 0.459 & - & - & NS \\
\hline Log Fasting blood glucose (mg/dL) & -0.179 & 0.319 & - & - & NS \\
\hline $\log \mathrm{HbAlc}(\%)$ & 0.117 & 0.512 & - & - & NS \\
\hline Log NT-proBNP (pg/mL) & -0.295 & 0.081 & - & - & NS \\
\hline \multicolumn{6}{|l|}{ Medicines } \\
\hline ARB/ACE-I use & -0.152 & 0.377 & - & - & NS \\
\hline$\beta$-Blocker use & -0.174 & 0.309 & - & - & NS \\
\hline$\alpha$-Blocker use & -0.145 & 0.399 & - & - & NS \\
\hline Calcium antagonist use & 0.454 & 0.005 & 0.387 & 0.532 & 0.006 \\
\hline Statin use & 0.465 & 0.004 & 0.322 & 0.558 & 0.024 \\
\hline
\end{tabular}

Variables with a $P<0.10$ on univariate analysis were incorporated into the multivariate models (adopted factors: systolic blood pressure, logPTX3, log-hsCRP, LDL-cholesterol, log-NT-proBNP, calicium antagonist use, and statin use). Abbreviations are same those in Table I. 
Table III. Correlation Between Change ratios of Flow Mediated Dilation (FMD) and Various Parameters

\begin{tabular}{lrc}
\hline \multicolumn{1}{c}{ Parameters } & $r$ & $P$ \\
\hline Systolic blood pressure (mmHg) & -0.126 & 0.473 \\
Diastolic blood pressure (mmHg) & 0.059 & 0.735 \\
Log PTX3 (pg/mL) & -0.446 & 0.006 \\
Log hsCRP (mg/L) & -0.330 & 0.050 \\
eGFR (mL/min/1.73m²) & 0.101 & 0.557 \\
Total cholesterol (mg/dL) & -0.032 & 0.852 \\
LDL-cholesterol (mg/dL) & -0.053 & 0.759 \\
HDL-cholesterol (mg/dL) & 0.022 & 0.899 \\
Log triglyceride (mg/dL) & 0.256 & 0.133 \\
Log fasting blood glucose (mg/dL) & -0.178 & 0.338 \\
Log HbA1c $(\%)$ & 0.137 & 0.446 \\
Log-NT-proBNP (pg/mL) & -0.319 & 0.058 \\
\hline
\end{tabular}

$r$ indicates Spearman's rank correlation coefficient. All other abbreviations are the same as those in Table I.

dothelial nitric oxide synthase (eNOS) activity. ${ }^{17-21)}$ In addition, CRP downregulates the number of endothelial progenitor cells and cell function in vitro. ${ }^{22}$ The long pentraxin PTX3 shares some similarities with CRP, but differs in terms of structural domain, gene organization, cellular and tissue sources, inducing stimuli, and recognized ligands. ${ }^{10)}$ PTX3 is produced at sites of inflammation, and it is intimately linked to endothelial dysfunction. ${ }^{23)}$ Gustin, et $a l^{24)}$ demonstrated that lysophosphatidic acid, a major bioactive lipid component of oxidized lowdensity lipoproteins, induces both PTX3 expression and mRNA in endothelial cells in vitro. Circulating endothelial progenitor cells levels mobilized by endothelial dysfunction are associated with PTX3 levels in patients with peripheral artery disease. ${ }^{25)}$ A histological study identified PTX3 positivity in the interstitium, the cytoplasm of macrophages, and the endothelium in heart tissue from patients with acute myocardial infarction. ${ }^{26)}$ These suggest that both PTX3 and CRP reflect endothelial function. The present study showed that plasma PTX3 is significantly correlated with endothelial function assessed by FMD in patients with clinically stable CAD. Furthermore, the change ratios of PTX3 and FMD also correlated, indicating that PTX3 levels are a potential biomarker for predicting endothelial function. Correlations between PTX3 levels and FMD are significantly negative in patients with type II diabetes without $\mathrm{CAD}^{27)}$ and chronic kidney disease. ${ }^{28)}$ Our study comprised patients with CAD, which means that plasma PTX3 levels can reflect endothelial function, even at an advanced stage of atherosclerosis. Indeed, immunohistochemical staining has shown intense PTX3 expression in humans with advanced atherosclerotic lesions. ${ }^{29)}$ Multivariate stepwise regression analysis in the present study showed that PTX3, but not hsCRP, is an independent factor associated with FMD. In addition, changes in FMD values correlated more closely with changes in levels of PTX3 than of hsCRP. This suggests that PTX3 levels reflect endothelial function more precisely than hsCRP levels, which agrees with the findings of another study on Turkish patients with type II diabetes and proteinuria. ${ }^{27)}$ This might be due to a difference in the synthetic processes and sites of synthesis between these two inflammatory markers. Collectively, plasma PTX3 levels might be more useful than serum hsCRP levels for predicting endothelial dysfunction.

Our study has several limitations. First, this was a retro-
A

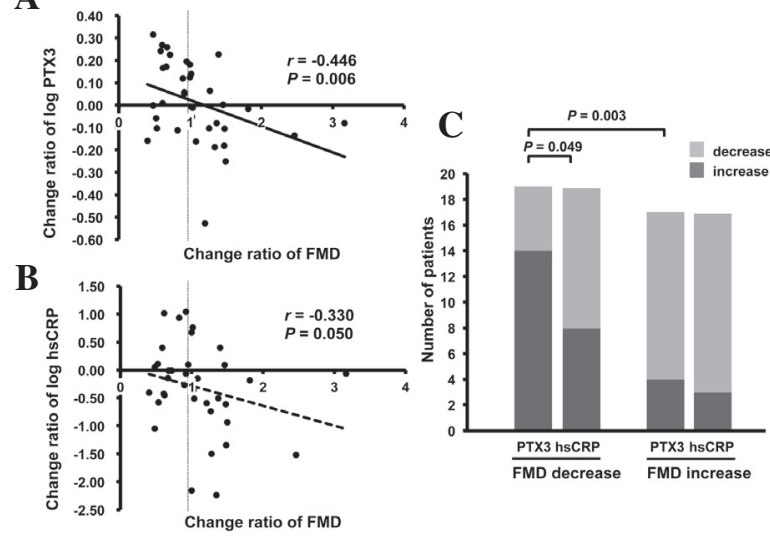

Figure 2. Association between changes in FMD and both PTX3 and hsCRP levels. Change ratios of FMD negatively correlate with change ratios of both log-PTX3 (A) and hsCRP (B). Comparison of both increased and decreased PTX3 and hsCRP levels between patients with either decreased or increased FMD (C). FMD indicates flow mediated dilation; hsCRP, high sensitive C-reactive protein; and PTX3, pentraxin 3.

spective analysis of a small patient cohort from a single center. Second, the enrolled patients had at least $\geq 75 \%$ stenosis in one coronary artery. However, the numbers, sites, and vulnerability of atherosclerotic lesions in both coronary and systemic arteries might have affected our results, although a complete evaluation of atherosclerosis is impossible in the clinical setting. Third, nitroglycerin-mediated vasodilation was not examined; therefore, endothelium-independent dilation remains unclear. Fourth, although all vasoactive medications were withheld for at least 12 hours prior to investigations, calcium antagonists might have affected the FMD values determined from the results of univariate and multivariate linear regression analyses. Regarding the effect of statins on FMD values, a meta-analysis has already revealed that statins can significantly improve endothelial dysfunction assessed by FMD in patients with diabetes. ${ }^{3)}$ Therefore, statins were associated with FMD values in the present study. Furthermore, differences in the duration of administration, dosage, and type of medicines were unable to be examined. Finally, whether PTX3 levels and FMD values are able to predict clinical outcomes was not addressed, and thus remains unclear. Future longitudinal and prospective studies of a large cohort are needed to address these issues.

Conclusion: Endothelial function assessed by FMD is associated with plasma PTX3 levels and their change ratios. In addition, this association is closer than that between hsCRP and endothelial function. These findings suggest that plasma PTX3 is a more potent predictor of endothelial function than hsCRP in patients with CAD.

\section{REFERENCES}

1. Ross R. Atherosclerosis -- an inflammatory disease. N Engl J Med 1999; 340: 115-26. (Review)

2. Furchgott RF, Vanhoutte PM. Endothelium-derived relaxing and contracting factors. FASEB J 1989; 3: 2007-18. (Review)

3. Zhang L, Gong D, Li S, Zhou X. Meta-analysis of the effects of statin therapy on endothelial function in patients with diabetes 
mellitus. Atherosclerosis 2012; 223: 78-85. (Review)

4. Suwaidi JA, Hamasaki S, Higano ST, Nishimura RA, Holmes DR Jr, Lerman A. Long-term follow-up of patients with mild coronary artery disease and endothelial dysfunction. Circulation 2000; 101: 948-54.

5. Neunteufl T, Heher S, Katzenschlager R, et al. Late prognostic value of flow-mediated dilation in the brachial artery of patients with chest pain. Am J Cardiol 2000; 86: 207-10.

6. Nawawi H, Osman NS, Annuar R, Khalid BA, Yusoff K. Soluble intercellular adhesion molecule-1 and interleukin-6 levels reflect endothelial dysfunction in patients with primary hypercholesterolaemia treated with atorvastatin. Atherosclerosis 2003; 169: 28391.

7. Araujo JP, Lourenco P, Azevedo A, et al. Prognostic value of highsensitivity C-reactive protein in heart failure: a systematic review. J Card Fail 2009; 15: 256-66. (Review)

8. Fichtlscherer S, Rosenberger G, Walter DH, Breuer S, Dimmeler S, Zeiher AM. Elevated C-reactive protein levels and impaired endothelial vasoreactivity in patients with coronary artery disease. Circulation 2000; 102: 1000-6.

9. Bottazzi B, Doni A, Garlanda C, Mantovani A. An integrated view of humoral innate immunity: pentraxins as a paradigm. Annu Rev Immunol 2010; 28: 157-83. (Review)

10. Deban L, Russo RC, Sironi M, et al. Regulation of leukocyte recruitment by the long pentraxin PTX3. Nat Immunol 2010; 11: 328-34.

11. Corretti MC, Anderson TJ, Benjamin EJ, et al. Guidelines for the ultrasound assessment of endothelial-dependent flow-mediated vasodilation of the brachial artery: a report of the International Brachial Artery Reactivity Task Force. J Am Coll Cardiol 2002; 39: 257-65.

12. Celermajer DS, Sorensen KE, Gooch VM, et al. Non-invasive detection of endothelial dysfunction in children and adults at risk of atherosclerosis. Lancet 1992; 340: 1111-5.

13. Matsuo S, Imai E, Horio M, et al. Revised equations for estimated GFR from serum creatinine in Japan. Am J Kidney Dis 2009; 53: 982-92.

14. Lusis AJ. Atherosclerosis. Nature 2000; 407: 233-41. (Review)

15. Cines DB, Pollak ES, Buck CA, et al. Endothelial cells in physiology and in the pathophysiology of vascular disorders. Blood 1998; 91: 3527-61. (Review)

16. Kullo IJ, Malik AR. Arterial ultrasonography and tonometry as adjuncts to cardiovascular risk stratification. J Am Coll Cardiol 2007; 49: 1413-26. (Review)

17. Hein TW, Singh U, Vasquez-Vivar J, Devaraj S, Kuo L, Jialal I. Human C-reactive protein induces endothelial dysfunction and uncoupling of eNOS in vivo. Atherosclerosis 2009; 206: 61-8.

18. Venugopal SK, Devaraj S, Yuhanna I, Shaul P, Jialal I. Demonstration that C-reactive protein decreases eNOS expression and bioactivity in human aortic endothelial cells. Circulation 2002; 106: 1439-41.

19. Singh U, Devaraj S, Vasquez-Vivar J, Jialal I. C-reactive protein decreases endothelial nitric oxide synthase activity via uncoupling. J Mol Cell Cardiol 2007; 43: 780-91.

20. Teoh H, Quan A, Lovren F, et al. Impaired endothelial function in C-reactive protein overexpressing mice. Atherosclerosis 2008; 201: $318-25$.

21. Guan H, Wang P, Hui R, Edin ML, Zeldin DC, Wang DW. Adenoassociated virus-mediated human C-reactive protein gene delivery causes endothelial dysfunction and hypertension in rats. Clin Chem 2009; 55: 274-84.

22. Verma S, Kuliszewski MA, Li SH, et al. C-reactive protein attenuates endothelial progenitor cell survival, differentiation, and function: further evidence of a mechanistic link between C-reactive protein and cardiovascular disease. Circulation 2004; 109: 205867.

23. Savchenko A, Imamura M, Ohashi R, et al. Expression of pentraxin 3 (PTX3) in human atherosclerotic lesions. J Pathol 2008; 215: 48-55.

24. Gustin C, Delaive E, Dieu M, Calay D, Raes M. Upregulation of pentraxin-3 in human endothelial cells after lysophosphatidic acid exposure. Arterioscler Thromb Vasc Biol 2008; 28: 491-7.

25. Morishita T, Uzui H, Nakano A, et al. Number of endothelial progenitor cells in peripheral artery disease as a marker of severity and association with pentraxin-3, malondialdehyde-modified lowdensity lipoprotein and membrane type-1 matrix metalloproteinase. J Atheroscler Thromb 2012; 19: 149-58.

26. Nebuloni M, Pasqualini F, Zerbi P, et al. PTX3 expression in the heart tissues of patients with myocardial infarction and infectious myocarditis. Cardiovasc Pathol 2011; 20: e27-35.

27. Suliman ME, Yilmaz MI, Carrero JJ, et al. Novel links between the long pentraxin 3, endothelial dysfunction, and albuminuria in early and advanced chronic kidney disease. Clin J Am Soc Nephrol 2008; 3: 976-85.

28. Yilmaz MI, Sonmez A, Ortiz A, et al. Soluble TWEAK and PTX3 in nondialysis CKD patients: impact on endothelial dysfunction and cardiovascular outcomes. Clin J Am Soc Nephrol 2011; 6: 785-92.

29. Rolph MS, Zimmer S, Bottazzi B, Garlanda C, Mantovani A, Hansson GK. Production of the long pentraxin PTX3 in advanced atherosclerotic plaques. Arterioscler Thromb Vasc Biol 2002; 22: e10-4. 